TITLE:

\title{
Expression of OATP1B3 determines uptake of Gd-EOB-DTPA in hepatocellular carcinoma
}

\section{AUTHOR(S):}

Narita, Masato; Hatano, Etsuro; Arizono, Shigeki; MiyagawaHayashino, Aya; Isoda, Hiroyoshi; Kitamura, Koji; Yasuchika, Kentaro; Nitta, Takashi; Uemoto, Shinji

\section{CITATION:}

Narita, Masato ...[et al]. Expression of OATP1B3 determines uptake of Gd-EOB-DTPA in hepatocellular carcinoma. Journal of Gastroenterology 2009, 44(7): 793-798

\section{ISSUE DATE:}

2009-07

URL:

http://hdl.handle.net/2433/85340

\section{RIGHT:}

c Springer 2009.; This is not the published version. Please cite only the published version.; この論文は出版社版でありません。引用の際には 出版社版をご確認ご利用ください。 


\title{
Expression of OATP1B3 determines uptake of Gd-EOB-DTPA in hepatocellular carcinoma
}

\author{
Masato Narita, MD* \\ Etsuro Hatano, MD, $\mathrm{PhD}^{*}$ \\ Shigeki Arizono, MD† \\ Aya Miyagawa-Hayashinoł \\ Hiroyoshi Isoda, MD, $\mathrm{PhD} \dagger$ \\ Koji Kitamura, MD* \\ Kojiro Taura, $\mathrm{MD}, \mathrm{PhD}^{*}$ \\ Kentaro Yasuchika, MD, PhD* \\ Takashi Nitta, MD, PhD* \\ Iwao Ikai, MD, PhD* \\ Shinji Uemoto, MD, PhD*
}

Department of Surgery* and Department of Diagnostic Imaging and Nuclear Medicine†, Graduate School of Medicine, Kyoto University

Department of Diagnostic Pathology, Kyoto University Hospital, Kyoto, Japanł 54 Shogoin-kawahara-cho, Sakyo-ku, Kyoto, 606-8507, Japan

\section{Correspondence and reprints request to}

Etsuro Hatano, MD, PhD

Department of Surgery, Graduate school of Medicine, Kyoto University

54 Shogoin-kawahara-cho, Sakyo-ku, Kyoto 606-8507, Japan

Telephone number: 81-75-751-4323, Fax number: 81-75-751-4348

E-mail: etsu@kuhp.kyoto-u.ac.jp

\section{Short title:}

Uptake of GD-EOB-DTPA in HCC 


\begin{abstract}
Background: Gadolinium-ethoxybenzyl-diethylenetriamine pentaacetic acid

(Gd-EOB-DTPA) is an MRI contrast agent with perfusion and hepatoselective properties.

The purpose of the study was to examine uptake of Gd-EOB-DTPA in the hepatobiliary phase in hepatocellular carcinoma (HCC).
\end{abstract}

Methods: A retrospective analysis of 22 patients with HCC who underwent preoperative Gd-EOB-DTPA-enhanced MRI was performed. Enhancement ratios (ERs) and expression levels of the organic anion transporter (OATP) 1B3 protein were examined.

Results: Gd-EOB-DTPA accumulated in the hepatobiliary phase in 6 of the 22 cases. All 6 Gd-EOB-DTPA-positive cases were moderately differentiated HCC, but 11 other moderately differentiated HCCs did not show Gd-EOB-DTPA uptake. Histopathologically, 4 Gd-EOB-DTPA-positive HCCs and $\underline{5}$ Gd-EOB-DTPA-negative HCCs produced bile. HCCs with Gd-EOB-DTPA uptake overexpressed OATP1B3 compared with HCCs without Gd-EOB-DTPA uptake, and OATP1B3 levels were significantly correlated with ERs (r=0.91, $\mathrm{P}<0.0001)$.

Conclusions: Uptake of Gd-EOB-DTPA in HCC is determined by expression of OATP1B3 rather than by tumor differentiation or bile production.

Keywords: Green hepatoma, Gd-EOB-DTPA, MRI, OATP1B3, HCC 


\section{Introduction}

In the past decade, several liver-specific magnetic resonance imaging (MRI) contrast media have been investigated in clinical studies with the goal of increasing the performance of liver MRI, especially for lesion detection. ${ }^{1}$ Gadolinium-ethoxybenzyl-diethylenetriamine pentaacetic acid (Gd-EOB-DTPA) is a recently developed liver-specific MRI contrast agent with combined perfusion and hepatocyte-selective properties. A bolus injection of Gd-EOB-DTPA provides a dual mode of action that allows imaging in the early dynamic phase (as with standard gadolinium chelates) and in the delayed phase (hepatobiliary phase) that begins 20 min after injection and lasts until 90 min after injection. ${ }^{2,3}$ In the hepatobiliary phase, hepatic lesions lacking normally functioning hepatocytes are imaged as a defect of hepatocyte-selective enhancement compared with normal parenchyma, ${ }^{4}$ and evaluation of vascularity and hepatocyte-specific uptake enables accurate detection and characterization of focal liver lesions. Therefore, Gd-EOB-DTPA-enhanced MRI is potentially superior to standard imaging using gadolinium chelates or spiral CT, especially for detection of small metastases and differential diagnosis of hypervascular lesions. ${ }^{5}$

A differential diagnosis of a dysplastic nodule and well-differentiated HCC can be based on findings with or without uptake of Gd-EOB-DTPA. ${ }^{6}$ However, some HCCs may show paradoxical uptake of Gd-EOB-DTPA and are recognized as iso- or hyperintense lesions in the hepatobiliary phase, compared with normal parenchyma. ${ }^{7,8}$ The characteristics of such Gd-EOB-DTPA-positive HCCs in the hepatobiliary phase has not been defined. Therefore, the aim of this study was to investigate the frequency of HCCs with uptake of Gd-EOB-DPTA in the hepatobiliary phase and to clarify the mechanism of uptake.

\section{Methods}

\section{Patients}


The study was performed as a retrospective review of patients with HCC who received Gd-EOB-DTPA-enhanced MRI as a preoperative examination and underwent liver resection at Kyoto University Hospital from May 2008 to October 2008. Patients who received pretreatment with transarterial chemoembolization (TACE) or percutaneous ethanol injection therapy (PEIT) in the preoperative period were excluded.

\section{MRI acquisition}

MR imaging was performed at 3 or $1.5 \mathrm{~T}$ using commercially available MR systems in the Magnetom series (Siemens, Erlangen, Germany): Magnetom Trio Tim (3 T), Magnetom Avanto (1.5 T) or Magnetom Symphony (1.5 T). All images were obtained in the axial plane. Dynamic and delayed hepatobiliary phase T1-weighted three-dimensional spoiled gradient echo images were obtained using a volumetric interpolated breath-hold examination (VIBE) sequence with chemically selective fat saturation (TR, 3 to 5.21 msec; TE, 1.06 to 2.13 msec; flip angle, $9^{\circ}$ to $15^{\circ}$; matrix, $205 \times 256,198 \times 320$ or $259 \times 320 \mathrm{~mm}$; field of view, $240 \times 320$ to $270 \times 360 \mathrm{~mm}$; number of signals acquired, 1 ; slice thickness, 3 to $5 \mathrm{~mm}$; slice number, 40 to 80 ; acquisition time, 14 to $22 \mathrm{sec}$ ). In pre-contrast imaging at $1.5 \mathrm{~T}$, parallel acquisition techniques were not used. In pre-contrast imaging at $3 \mathrm{~T}$ and in post-contrast imaging, iPAT reconstruction was performed using the GRAPPA algorithm with a parallel acquisition technique factor of two. Parameters varied according to the machines used and the patient physique. Images with the initial VIBE sequence were acquired before administration of the contrast agent. A bolus of $25 \mu \mathrm{mol} / \mathrm{kg}$ body weight Gd-EOB-DTPA (Bayer, Germany) was then injected and flushed with $40 \mathrm{ml}$ of sterile saline solution from the antecubital vein. A power injector (Sonic Shot, Nemoto-Kyorindo, Tokyo, Japan) was used for injection of the contrast agent and saline. After the dynamic study, images were obtained with a T1-weighted VIBE sequence in the delayed hepatobiliary phase at 20-25 min after injection of the contrast agent. 


\section{Image analysis}

The enhancement ratio (ER) of each lesion diagnosed as HCC was calculated according to Saito et al. ${ }^{8}$ Briefly, the signal intensity (SI) of the lesion was measured on T1-weighted images collected before injection of the contrast agent and 20-25 min after the injection. The SI of the tumor was measured by defining an oval region of interest (ROI) that covered as much of the lesion as possible. ERs were calculated with the formula: ER $=[(\mathrm{SI}$ after enhancement-SI before enhancement)/SI before enhancement].

\section{Evaluation of bile production}

Bile production was evaluated blindly by a liver-specialized pathologist (A. M-H.) using hematoxylin and eosin (HE) staining. Positive bile production was indicated by the presence of bile pigment inside the pseudo-cholangiole, bile canaliculi, or cytoplasm of tumor cells. ${ }^{9}$ To perform semi-quantitative evaluation of bile production, bile production was stratified into 3-scale according to the degree of it: Mild (<5\%); Moderate (5-25\%); Severe ( $>25 \%)$. Western blot analysis

Tissue samples were homogenized in lysis buffer containing $50 \mathrm{mM}$ Tris- $\mathrm{HCl}$ (pH 6.8), $10 \%$ glycerol and $2 \%$ sodium dodecylsulfate. After the concentration of the sample was determined, 0.1\% bromophenol blue and 5\% 2-mercaptoethanol was added. For immunoblotting, protein $(24 \mu \mathrm{g})$ was subjected to SDS-PAGE and then transferred to a polyvinylidene difluoride membrane. The membranes were blocked with Blocking-One (Nacalai Tesque, Kyoto, Japan) and incubated with a primary antibody against OATP1B3 (\#651140; Progen Biotechnik, Heidelberg, Germany) at a dilution of $6 \mu \mathrm{g} / \mathrm{mL}$ in Blocking-One or an anti-actin antibody (\#sc-1615; Santa Cruz Biotechnology, Santa Cruz, CA, USA) at 1:1000 dilution overnight at $4^{\circ} \mathrm{C}$. After washing, membranes were reacted with horseradish peroxidase-conjugated antibodies (Santa Cruz). Chemiluminescence was detected with Immobilon Western HRP Substrate (Millipore, Billerica, MA, USA) and the 
intensity of the bands was quantified with Quantity One imaging analysis software (Bio-Rad Laboratories, Hercules, CA, USA).

Immunohistochemistry

For immunohistochemistry of OATP1B3, paraffin sections were pretreated with $0.3 \%$ $\underline{\mathrm{H}}_{2} \underline{\mathrm{O}}_{2}$ in methanol and then subjected to antigen retrieval in citrate buffer (10 mM, pH 6.0) in a pressure cooker. After blocking with 3\% BSA-10\% normal serum for $1 \mathrm{~h}$, the sections were incubated with a primary antibody recognizing OATP1B3 (Progen Biotechnik) overnight at $\underline{4^{\circ} \mathrm{C} \text {. Subsequently, the sections were incubated with Labeled Polymer in an Envision + }}$ System HRP Kit (Dako, Tokyo, Japan) at room temperature for $1 \mathrm{~h}$. The sections were examined after incubation with a Liquid DAB Substrate Chromogen System (Dako) and counterstained with hematoxylin.

Statistical analysis

Statistical analysis was performed using SPSS v. 11.0.1 (SPSS Inc., Chicago, IL, USA). Data are expressed as means \pm SD. Differences in measured variables between each group were assessed using a Mann-Whitney $U$ test with $P<0.05$ considered to indicate statistical significance. Pearson correlation analysis was used to evaluate the relationship between ERs and expression levels of OATP1B3 protein, with a correlation coefficient $(r)$ of $\geq 0.8$ indicating a strong correlation.

\section{Results}

From May 2008 to October 2008, 30 consecutive patients with HCCs underwent liver resection at Kyoto University Hospital. Five of these patients did not receive preoperative Gd-EOB-DTPA-enhanced MRI and 3 were treated with TACE $(n=3)$ and PEIT $(n=1)$ before surgery, leaving 22 patients for evaluation. No hepatocyte-specific enhancement in Gd-EOB-DTPA-enhanced MRI in the hepatobiliary phase was observed in 16 cases (Figs. 
1A, B), whereas hyperintensive lesions were found in 6 cases (Figs. 1C, D). The patients were divided into two groups based on the ER (Table 1) and all cases with high ER ( $\geq 1)$ were found to be moderately differentiated HCCs. Macroscopically, HCCs producing bile were identified by a greenish color in HE staining; so-called green hepatoma (Fig. 2). HCCs with bile production were observed in $\underline{5}$ of 16 cases $(\underline{31.3 \%})$ with a low ER $(<1)$ and 4 of 6 cases $(66.7 \%)$ with a high ER $(P=0.231)$. In the low ER group, the details of the bile production in 5 cases were described as follows: Mild; 2, Moderate; 1 , and Severe; 2 . On the other hand, all cases diagnosed as bile production HCCs were stratified into the Mild in the high ER group.

Oatp1 is involved in gadoxetate uptake into rat liver ${ }^{10}$ and therefore expression of OATP1B3 was examined in tumor and corresponding non-tumor tissues by Western blotting. Non-tumor liver parenchyma were diagnosed as normal parenchyma $(n=4)$, chronic hepatitis $(n=6)$, liver cirrhosis $(n=8)$, liver fibrosis $(n=3)$, and steatosis $(n=1)$. There was no significant difference in non-tumor liver tissues. On the other hand, OATP1B3 was hardly detectable in HCCs with a low ER, but strongly expressed in HCCs with a high ER ( $<$ 0.0001; Fig. 3A). Immunohistochemical analysis indicated that OATP1B3 was highly expressed in tumor cell membrane and weakly expressed in tumor cytoplasm in HCCs with a high ER (Fig. 3B). In contrast, expression of OATP1B3 was hardly observed in HCCs with a low ER (Fig. 3C). Furthermore, ERs were significantly correlated with OATP1B3 levels ( $r=$ 0.91, $P<0.0001$; Fig. 3ㅁ).

\section{Discussion}

The mechanism of uptake of Gd-EOB-DTPA in HCCs and the characteristics of Gd-EOB-DTPA-positive HCCs have not been clarified to date. In the present study, 6 of 22 HCCs (27.3\%) accumulated Gd-EOB-DTPA in the hepatobiliary phase. This raises questions 
regarding the kind of HCC that accumulates Gd-EOB-DTPA and the clinical importance of uptake of Gd-EOB-DTPA. Well-differentiated HCCs have been shown to be more enhanced than the surrounding liver parenchyma in the hepatobiliary phase, suggesting that MRI with Gd-EOB-DTPA can be used to predict the extent of differentiation of tumors ${ }^{11}$, but it has also been suggested that positive enhancement does not depend on tumor differentiation ${ }^{10}$. These studies were both performed in animals and the situation in human HCC remains unclear. In the current study, uptake of Gd-EOB-DTPA in the hepatobiliary phase was only observed in moderately differentiated HCCs. However, 11 other moderately differentiated HCCs did not accumulate Gd-EOB-DTPA. The frequency of bile production in HCCs with a high ER was similar to that of HCCs with a low ER. These results suggest that neither tumor differentiation nor bile production are correlated with Gd-EOB-DTPA enhancement in MRI in HCCs in the hepatobiliary phase.

OATPs are sodium-independent organic anion transporters that are expressed in many tissues, including the liver, kidney, intestine and brain. OATPs are involved in transport of a wide range of amphipathic organic anions, including bile salts, thyroid hormones, steroid hormones and their conjugates, as well as organic cations such as $\mathrm{N}$-methylquinidine. ${ }^{12}$ In addition to endogenous compounds, OATPs are capable of transporting xenobiotics and drugs, and therefore play an important role in drug absorption, disposition and excretion. OATP1B3, which is expressed in the human liver in the basolateral membrane of hepatocytes, is especially important for transport of agents such as Gd-EOB-DTPA, as well as uptake of endogenous substances and xenobiotics into hepatocytes. ${ }^{2,12-14}$ Gd-EOB-DTPA is transported into hepatocytes via OATPs and excreted into bile canaliculi via the multidrug resistance-associated protein MRP2. ${ }^{15}$

OATP1B3 protein was overexpressed in all HCCs that showed accumulation of Gd-EOB-DTPA in the hepatobiliary phase, whereas almost no expression of OATP1B3 was 
observed in most HCCs that did not show uptake of Gd-EOB-DTPA. Furthermore, a strong correlation was observed between ERs and expression levels of OATP1B3 protein. We also examined the expression of MRP2 protein by immunoblot analysis, since this protein is another transporter of bile salts ${ }^{16}$, but expression of MRP2 was neither correlated with ERs nor bile production (data not shown). It is of note that OATP1B3 also transports anticancer drugs such as the antifolate methothrexate, ${ }^{17}$ and paclitaxel and docetaxel. ${ }^{18}$ Therefore, HCC with upregulation of OATP1B3 may take up these drugs as well as Gd-EOB-DTPA, and this suggests that preoperative Gd-EOB-DTPA-enhanced MRI can be used to predict the potential efficacy of anticancer drugs transported by OATP1B3.

In conclusion, our results suggest that expression of OATP1B3 determines the uptake of Gd-EOB-DTPA in the hepatobiliary phase in HCCs, rather than tumor differentiation or bile production. However, the study is limited by the small number of cases and a further study is needed to confirm the correlation between expression of OATP1B3 and Gd-EOB-DTPA uptake and the clinical significance of Gd-EOB-DTPA enhancement in MRI of HCCs. 


\section{References}

1. Semelka RC, Helmberger TK. Contrast agents for MR imaging of the liver. Radiology 2001;218:27-38.

2. Hamm B, Staks T, Muhler A, Bollow M, Taupitz M, Frenzel T, et al. Phase I clinical evaluation of Gd-EOB-DTPA as a hepatobiliary MR contrast agent: safety, pharmacokinetics, and MR imaging. Radiology 1995;195:785-92.

3. Vogl TJ, Kummel S, Hammerstingl R, Schellenbeck M, Schumacher G, Balzer T, et al. Liver tumors: comparison of MR imaging with Gd-EOB-DTPA and Gd-DTPA. Radiology 1996;200:59-67.

4. Reimer P, Rummeny EJ, Shamsi K, Balzer T, Daldrup HE, Tombach B, et al. Phase II clinical evaluation of Gd-EOB-DTPA: dose, safety aspects, and pulse sequence. Radiology 1996;199:177-83.

5. Zech CJ, Herrmann KA, Reiser MF, Schoenberg SO. MR imaging in patients with suspected liver metastases: value of liver-specific contrast agent Gd-EOB-DTPA. Magn Reson Med Sci 2007;6:43-52.

6. Ichikawa T. MRI in the evaluation of hepatocellular nodules: role of pulse sequences and contrast agents. Intervirology 2004;47:252-70.

7. Huppertz A, Haraida S, Kraus A, Zech CJ, Scheidler J, Breuer J, et al. Enhancement of focal liver lesions at gadoxetic acid-enhanced MR imaging: correlation with histopathologic findings and spiral CT--initial observations. Radiology 2005;234:468-78.

8. Saito K, Kotake F, Ito N, Ozuki T, Mikami R, Abe K, et al. Gd-EOB-DTPA enhanced MRI for hepatocellular carcinoma: quantitative evaluation of tumor enhancement in hepatobiliary phase. Magn Reson Med Sci 2005;4:1-9.

9. $\quad$ Scheuer PJ, Lefkowitch JH. Liver Biopsy Interpretation, 7th ed, 2006 WB Saunders. 
10. Fujita M, Yamamoto R, Takahashi M, Tsuji T, Yamanaka T, Miyazawa T, et al. Paradoxic uptake of Gd-EOB-DTPA by hepatocellular carcinoma in mice: quantitative image analysis. J Magn Reson Imaging 1997;7:768-70.

11. Ni Y, Marchal G, Yu J, Muhler A, Lukito G, Baert AL. Prolonged positive contrast enhancement with Gd-EOB-DTPA in experimental liver tumors: potential value in tissue characterization. J Magn Reson Imaging 1994;4:355-63.

12. Konig J, Seithel A, Gradhand U, Fromm MF. Pharmacogenomics of human OATP transporters. Naunyn Schmiedebergs Arch Pharmacol 2006;372:432-43.

13. van Montfoort JE, Stieger B, Meijer DK, Weinmann HJ, Meier PJ, Fattinger KE. Hepatic uptake of the magnetic resonance imaging contrast agent gadoxetate by the organic anion transporting polypeptide Oatp1. J Pharmacol Exp Ther 1999;290:153-7.

14. Libra A, Fernetti C, Lorusso V, Visigalli M, Anelli PL, Staud F, et al. Molecular determinants in the transport of a bile acid-derived diagnostic agent in tumoral and nontumoral cell lines of human liver. J Pharmacol Exp Ther 2006;319:809-17.

15. Pascolo L, Petrovic S, Cupelli F, Bruschi CV, Anelli PL, Lorusso V, et al. Abc protein transport of MRI contrast agents in canalicular rat liver plasma vesicles and yeast vacuoles. Biochem Biophys Res Commun 2001;282:60-6.

16. Borst P, Elferink RO. Mammalian ABC transporters in health and disease. Annu Rev Biochem 2002;71:537-92.

17. Abe T, Unno M, Onogawa T, Tokui T, Kondo TN, Nakagomi R, et al. LST-2, a human liver-specific organic anion transporter, determines methotrexate sensitivity in gastrointestinal cancers. Gastroenterology 2001;120:1689-99.

18. Smith NF, Acharya MR, Desai N, Figg WD, Sparreboom A. Identification of OATP1B3 as a high-affinity hepatocellular transporter of paclitaxel. Cancer Biol 
Ther 2005;4:815-8. 
Table 1. Comparison of characteristics between patients with HCCs with ER $\geq 1$ and $<1$

\begin{tabular}{|c|c|c|c|}
\hline & $\begin{array}{l}E R \geq 1 \\
(n=6)\end{array}$ & $\begin{array}{c}\mathrm{ER}<1 \\
(\mathrm{n}=16)\end{array}$ & $P$ value \\
\hline \multicolumn{4}{|l|}{ Sex } \\
\hline Male & 5 & 1 & \multirow{2}{*}{0.91} \\
\hline Female & 1 & 3 & \\
\hline Age & $58.0 \pm 10.4$ & $62.3 \pm 11.5$ & 0.37 \\
\hline \multicolumn{4}{|l|}{ Histological grade } \\
\hline Well differentiated & 0 & 3 & \\
\hline Moderately differentiated & 6 & 11 & 0.294 \\
\hline Poorly differentiated & 0 & 2 & \\
\hline Tumors with bile production & 4 & $\underline{5}$ & $\underline{0.231}$ \\
\hline Mild $(<5 \%)$ & $\underline{4}$ & $\underline{2}$ & \\
\hline Moderate (5-25\%) & $\underline{0}$ & $\underline{1}$ & \\
\hline Severe $(>25 \%)$ & $\underline{0}$ & $\underline{2}$ & \\
\hline OATP1B3 / Actin expression ratio & $1.34 \pm 0.59$ & $0.34 \pm 0.29$ & $<0.001$ \\
\hline
\end{tabular}




\section{Figure Legends}

Figure 1. (A and B) MRI images of HCC without hepatocyte-selective enhancement in a 56-year-old-woman with a normal liver. Arrows indicate HCC located in a posterior liver segment. (C and D) MRI images of HCC with hepatocyte-selective enhancement in a 67-year-old-man with mild steatosis. Arrows indicate HCC located in a posterior liver segment.

Figure 2. (A) Surgically removed specimen. The cut surface of the tumor has a greenish color (green hepatoma). (B) HE staining of HCC (400x). The arrow heads indicate bile production on the insides of cytoplasm of tumor cells.

Figure 3. (A) Western blot for OATP1B3 and Actin (control) and densitometry ratio of the OATP1B3 level to that of the control. Non-tumor liver parenchyma were diagnosed as normal parenchyma $(n=4)$, chronic hepatitis $(n=6)$, liver cirrhosis $(n=8)$, and there was no significant difference in non-tumor liver tissues. On the other hand, expression of OATP1B3 was significantly higher in HCCs with ER $\geq 1$ compared to HCCs with ER $<1$. Liver sections from HCC with a high ER (B) and a low ER (C) were subjected to immunohistochemical analysis to determine expression patterns of OATP1B3 protein. Expression of OATP1B3 was observed predominantly in tumor cell membrane in HCC with a high ER. (D) A strong correlation was observed between expression levels of OATP1B3 and ERs. 
Figure 1

Pre-injection of Gd-EOB-DTPA

A

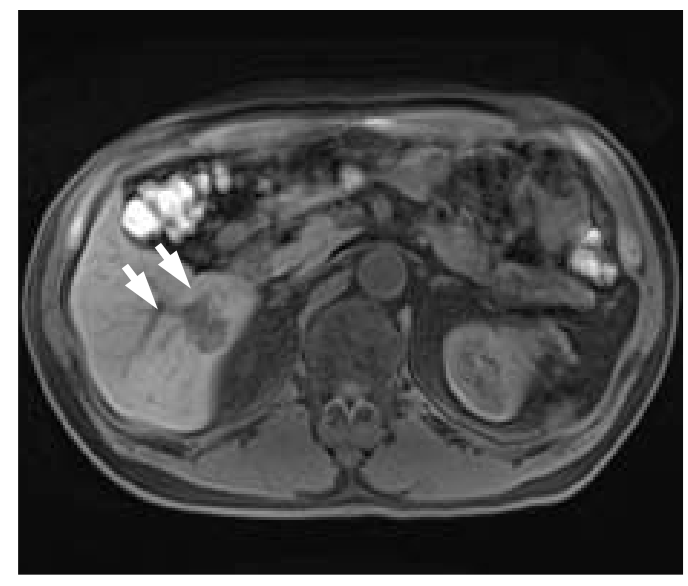

C

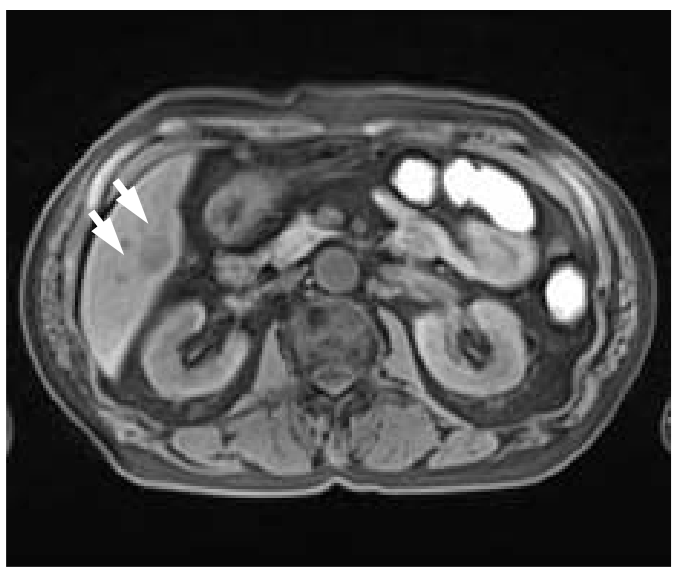

Post-injection of Gd-EOB-DTPA

B

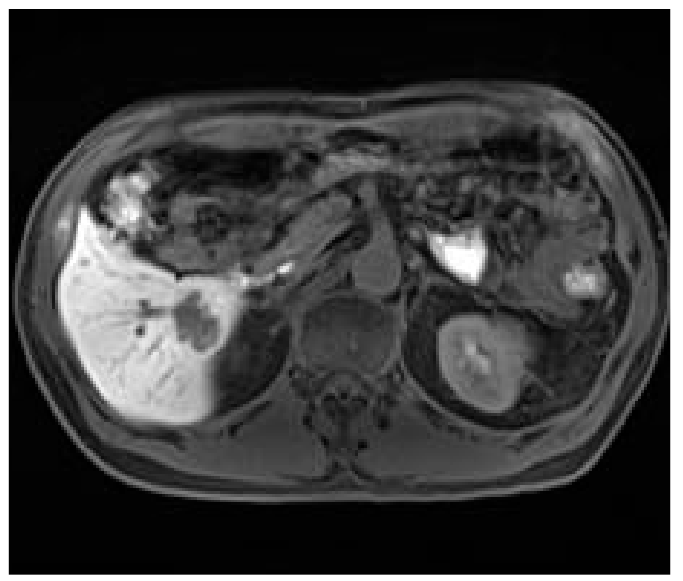

D

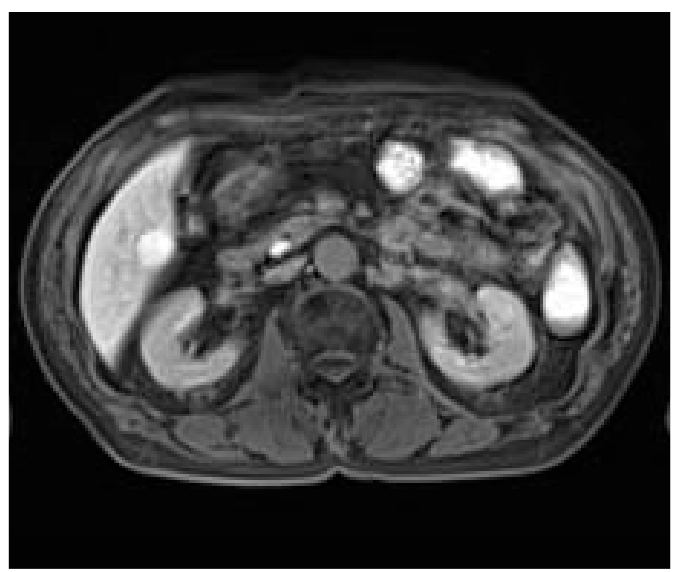




\section{Figure 2}
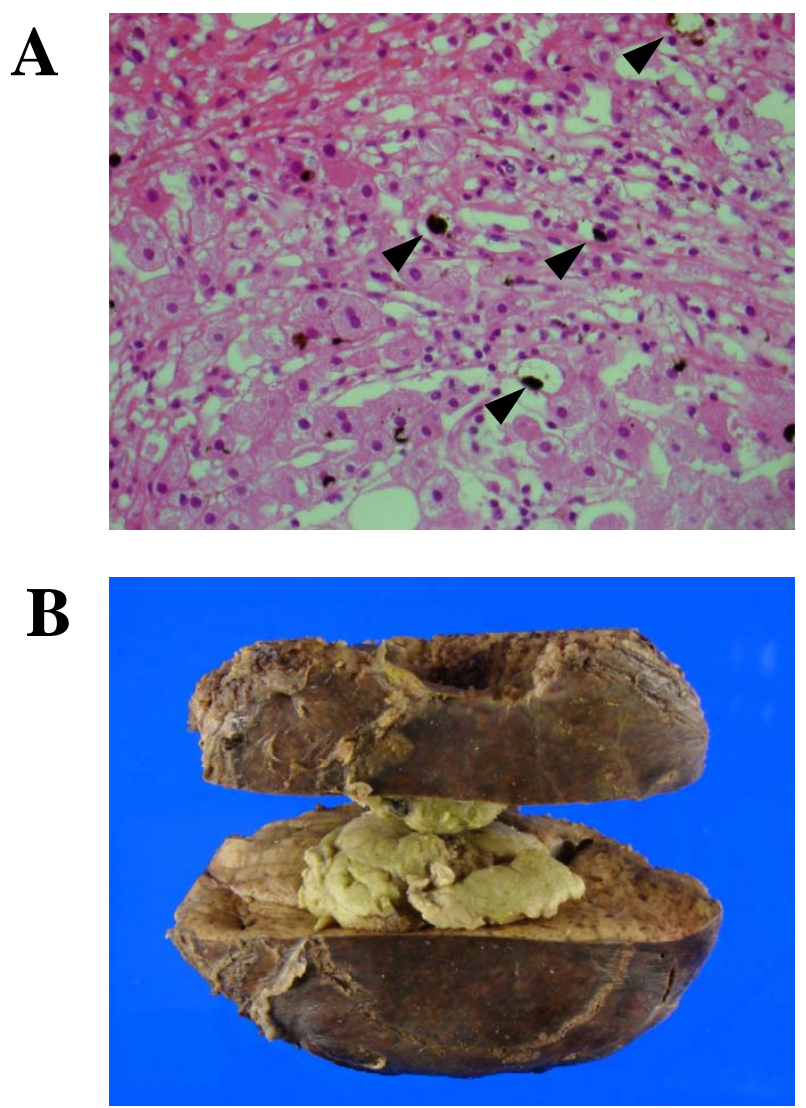


\section{Figure 3}

A
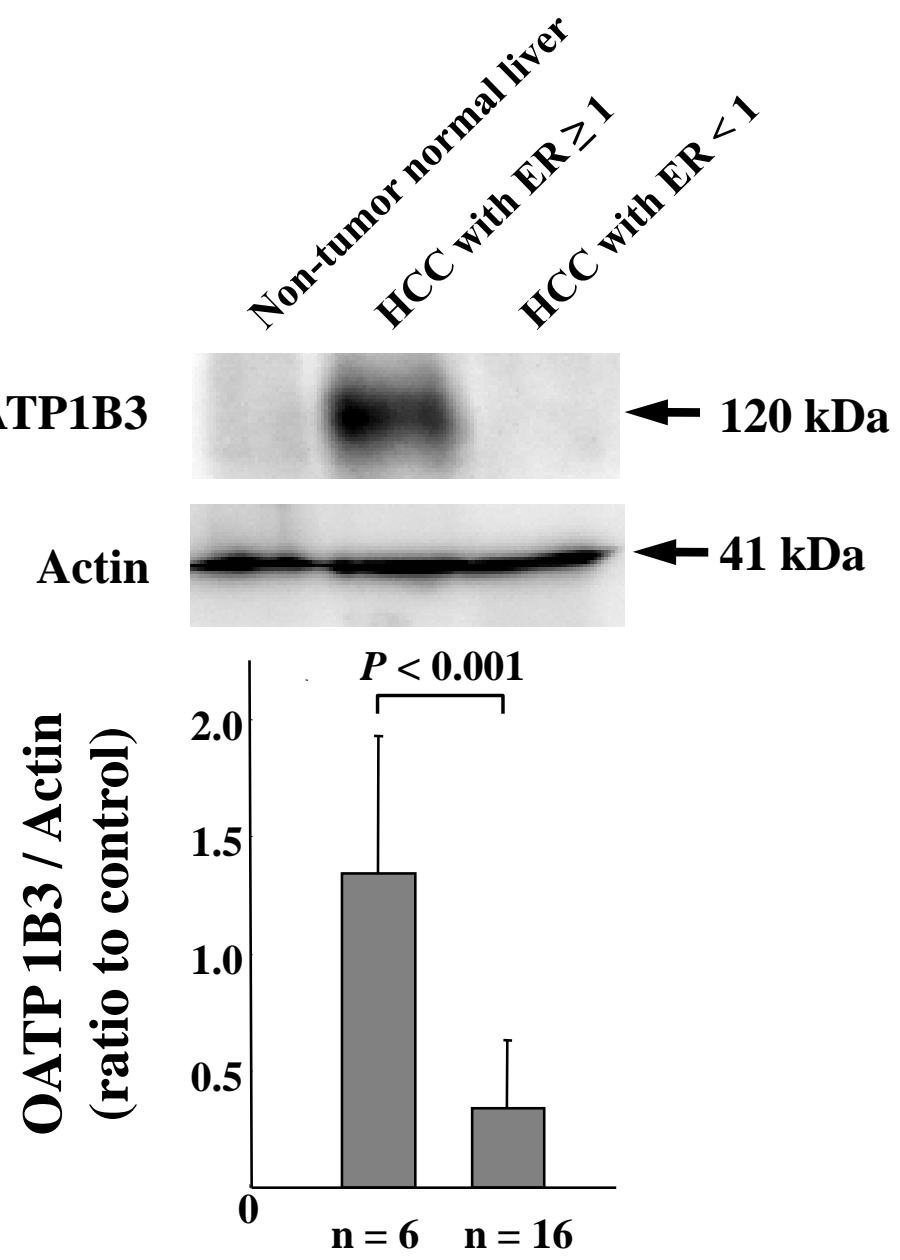

B

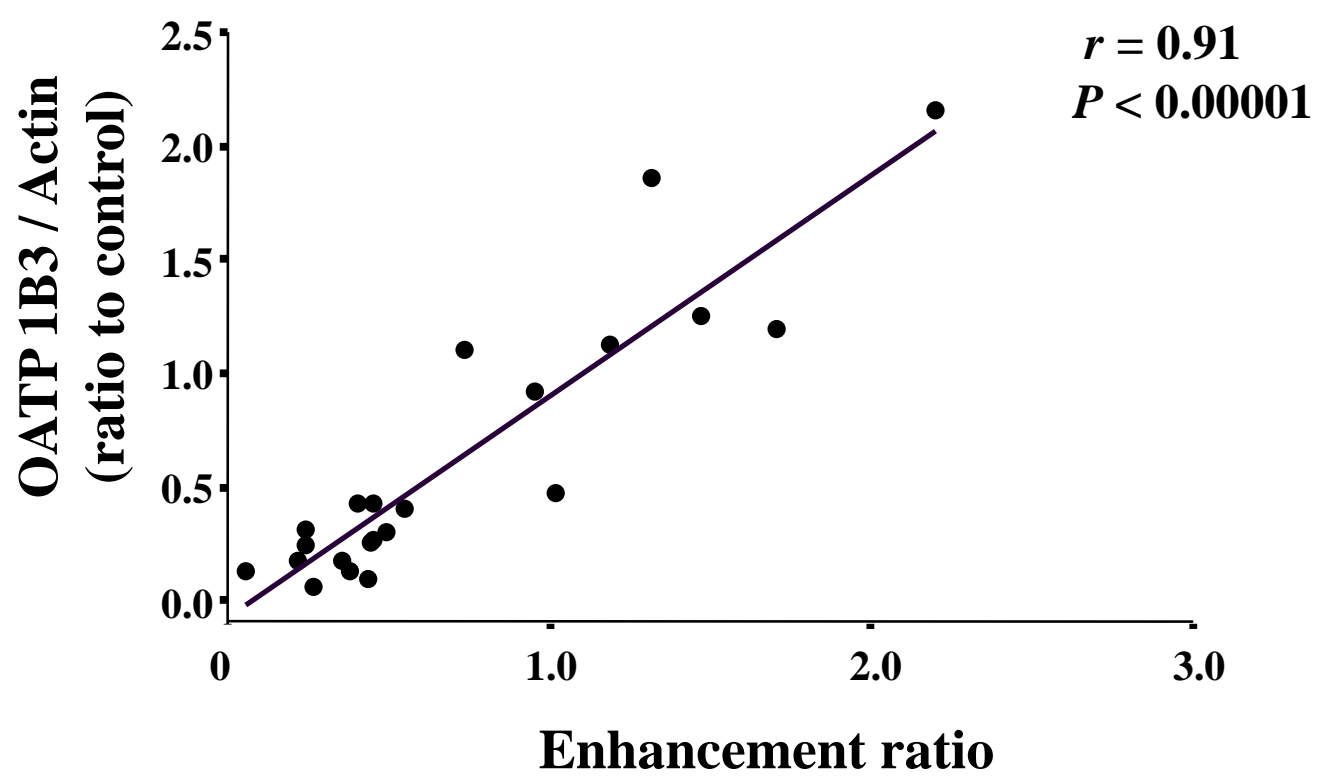

